\title{
Dynamic Routing Mechanism in Network Simulation Based on Topology Perception Time Series \\ Lei-gang SUN ${ }^{1, *}$, Yu-feng MA ${ }^{1}$, Li-ju $\mathrm{CHEN}^{1}$ and Bo WU ${ }^{1}$ \\ ${ }^{1}$ Xi'an Communication Institute, Changan District, Xi'an, China \\ *sunleigang0520@163.com
}

Keywords: Network simulation, Dynamic routing, Perception time, Routing algorithm.

\begin{abstract}
A fundamental requirement for any routing mechanism in network simulation is to truly reflect the forwarding process of packet from source to destination in the simulated topology. In current network simulators, all the nodes know the information instantaneously when the topology changed, which is obviously unrealistic, serious time distortion problem existed. Analyzed the static neighbor index vector mechanism, an abstract model of dynamic routing was presented, and an approach was proposed to compute the time needed for each node to know the topology changes. Dynamic routing lookup algorithm was designed, which maintained static routing table and a message queue of topology changes, and lookup routing states according to the perception time of topology changes, reduced the time consumption during route calculation. Experimental results show that our approach can improve the realism of dynamic routing in network simulation, and with high simulation efficiency.
\end{abstract}

\section{Introduction}

In the real network environment, the basic purpose of using dynamic routing protocol is to transmit information when the network topology changes, the nodes update routing table relying on the adaptive ability to network topology changes. In network simulation, in order to achieve the correct routing of the simulated packets, we can simulate the routing protocols with high authenticity, such as C-BGP, EIGRP, OSPF and RIP[1,2], this method is usually used for the development and verification of network protocols, but simulating complex routing protocols completely and accurately is very difficult to achieve, with high requirements for hardware devices and low simulation efficiency.

In most cases, the research of network simulation has more emphasis on network behavior, the implementation of all the details of the routing protocol is not necessary, the strategy commonly used is still using the static centralized routing table storage and search strategy in the network simulator, when the network topology changes, it reconstructs and updates the centralized routing table according to a new strategy, so as to adapt to the change of the routing information caused by the dynamic changes of network topology. HAO proposed a routing storage strategy based on the minimum spanning tree in reference[3]. Reference[4] proposed a method to calculate the dynamic routing of network simulation in real-time.

This paper aims to solve the authenticity and performance problems in the process of dynamic routing simulation. On the basis of the analyzing of static neighbor index vector routing strategy[5], taking perception time of different nodes into account, the paper proposed a dynamic routing strategy model in network simulation, analyzed and solved the two key technologies in the process of realization of the strategy: determine the perception time during topology change and design dynamic routing lookup algorithm. In this strategy, static routing table and topology change perception series 
form the complete topology of the simulation network, the routing table lookup is realized by comparing perception time of the nodes.

\section{Static Neighbor Index Vector Routing Strategy}

Time complexity and space complexity are two important properties that mutually contradictory of the local static routing strategy. Riley[6] proposed a routing strategy based on neighbor index vector, which is a better solution to solve the static routing problem in time and space, and is widely used in the current popular network simulator, such as OPNET, NS2, OMNeT++[7]. The basic principle is: we will not compute and storage routing information before simulation, the required storage space for routing table is 0 . Simulation packets generated with the simulation starting, routing table will be calculated in real-time according to the source address and destination address, the calculated routing information will be stored in the form of neighbor index vector in the packet header, when the packet arrives a routing node, we can realize the correct routing by extracting and analyzing the neighbor index vector contained in the header.

The static neighbor index vector routing strategy is described as follows: if the neighbor routing node number of a routing node is $M$, we can use a $k=\left\lceil\log _{2}^{M}\right\rceil$ bits binary string to represent the $M$ neighbor nodes, the method is: set this node as the origin node, firstly, marker the edge weight as 0 which connected to this origin node. Secondly, in accordance with the clockwise direction, mark other neighbor nodes increasing as $1,2,3, \ldots, \mathrm{M}-1$, mark a node as 0 if the node doesn't have a neighbor node, all the mark information stored in the cache. Thirdly, connect all the binary adjacent relation as a path $P$, form the neighbor index vector from source node to destination node of this path, the vector is encapsulated in the header of the simulated packet, when the packet reach the routing node, extract the first $k=\left\lceil\log _{2}^{M}\right\rceil$ bits of the adjacent index vector as search index, then we can determine the packet's next routing hop in the neighbor nodes, the data packet routing completed.

Neighbor index vector routing strategy computes routes during simulation process in real-time, the routing table storage space requirements is 0 before the beginning of the simulation, thus avoid the use of static routing table, save storage space greatly, and expand the size of the simulation. In a network topology with a number of nodes and edges are $\mathrm{n}$ and $\mathrm{e}$, describe the network topology by adjacent list, the routing lookup time complexity of the network is $O(n+e)$.

\section{Dynamic Routing Mechanism Based on Topology Perception Rime Aeries}

This section proposes the abstract model of dynamic routing, gives the calculation method to compute the time needed for each node to know the topology changes based on static neighbor index vector strategy, makes the simulation more closer to the real situation; the dynamic routing lookup algorithm is designed that can effectively reduce the cost of the route calculation and improve simulation efficiency. 


\section{Abstract Model of Dynamic Routing for Network Simulation}

Provided the initial topology is $G\left(V_{t_{0}}, E_{t_{0}}\right)$ for network topology $G(V, E)$ in time $t_{0}$, and the simulation time is set to $n s$. Assumed that in the simulation process, network topology changed $m$ times in time $t_{1}, t_{2}, \ldots t_{m}\left(0<t_{1}<t_{2}<\cdots<t_{m}<n\right)$, and each topology change time $t_{i}$ corresponding to a network topology $G\left(V_{t_{i}}, E_{t_{i}}\right)$. Therefore, these network topologies form an ordered network topology set $\left\{G\left(V_{t_{1}}, E_{t_{1}}\right), G\left(V_{t_{2}}, E_{t_{2}}\right), \cdots, G\left(V_{t_{m}}, E_{t_{m}}\right)\right\}$ in the $\mathrm{m}$ times process of change, and each topology corresponds to a shortest path routing table that is $\left\{R T_{t_{1}}, R T_{t_{2}}, \cdots, R T_{t_{m}}\right\}$.

The perception time of the nodes to know the topology that changes dynamically is defined as:

Definition 1 (perception time). For any node in the simulation network, when it is informed that a particular network topology changed, until the process of its own routing table updates the information completed, calls perception time of node to topology change.

A node in a network topology in time $t_{i}$ correspond to $G\left(V_{t_{i}}, E_{t_{i}}\right)$, supposes the node to perceive the next time for topology changes is $t_{i+1}$, and the corresponding network topology is $G\left(V_{t_{i+1}}, E_{t_{i+1}}\right)$, so the routing tables taken by the simulation network is $R T_{t_{j}}$ at time $\left[t_{i}, t_{i+1}\right)$. Due to a certain delay for perception time of each routing node or a source packet node to network topology information in the network, so there should be $t_{i}>t_{j}$.

According to the description above, the objective of dynamic routing lookup in network simulation is: the routing table $R T_{t_{j}}$ was used for node a (a routing node or packet source node) within $t_{k} \in\left[t_{i}, t_{i+1}\right)$ at any time, a packet $p_{k}$ sent from node a (the source address is $s r c$, and the destination address is $d s t$ ) can calculate the next-hop routing node NextHop $_{t_{k}, p_{k}, a}$ through dynamic routing lookup algorithm (Dynamic Routing Lookup: DRL), the abstract model can be expressed as:

$$
\operatorname{NextHop}_{t_{k}, p_{k}, a}=\operatorname{DRL}\left(\operatorname{src}, d s t, a, R T_{t_{j}}\right)
$$

Based on Eq. 1, the two key issues to be resolved in dynamic routing strategy are: 1) determine routing table $R T_{t_{i}}$ used by node a at any time in $\left.t_{k} \in\left[t_{i}, t_{i+1}\right) ; 2\right)$ design of DRL dynamic routing lookup algorithm.

\section{Determination of Topology Perception Time}

Ordered set of topology changes $\left\{G\left(V_{t_{1}}, E_{t_{1}}\right), G\left(V_{t_{2}}, E_{t_{2}}\right), \cdots, G\left(V_{t_{m}}, E_{t_{m}}\right)\right\}$ and the updated routing table $\left\{R T_{t_{1}}, R T_{t_{2}}, \cdots, R T_{t_{m}}\right\}$ are correspondence at any time in $\left\{t_{1}, t_{2}, \cdots, t_{m}\right\}$, so the perception time of node $a$ uniquely identifies the corresponded routing table $R T_{t_{j}}$ in $t_{k} \in\left[t_{i}, t_{i+1}\right)$. Through calculating perception time of node $a$ for each network topology change, the corresponding topology information can be determined, so the corresponded routing table $R T_{t_{j}}$ can also be determined.

In real network, when the network topology changes are caused by the failure or recovery of edge $e$, two nodes $n_{1}$ and $n_{2}$ of connecting the edges will be the first two to perceive this change, and the perception time $t_{0}$ is determined by the routing protocol 
the network used. Then, the network will widely search the entire network from $n_{1}$ and $n_{2}$, and notice the topology change information to every nodes in the network. Assume that nodes $n_{i-1}, n_{i}$ are two neighbor nodes in the network, and the perception time for the network topology changes is $t_{i-1}$ and $t_{i}$, there is the following formula:

$$
t_{i}=t_{i-1}+t_{T\left(n_{i-1}, n_{i}\right)}+t_{D\left(n_{i-1}, n_{i}\right)}+t_{C\left(n_{i-1}, n_{i}\right)}
$$

Where: $t_{T\left(n_{i-1}, n_{i}\right)}$ is forward time of packets routing, and $L$ indicates the average length of routing information in network protocol, and Datarate is the data rate of routing node, and there is

$t_{T\left(n_{i-1}, n_{i}\right)}=L /$ Datarate

$t_{D\left(n_{i-1}, n_{i}\right)}$ is the packet transmission time from node $n_{1}$ to $n_{2}$. If the connection bandwidth between nodes is $B_{n 1, n 2}$, there is

$$
t_{D\left(n_{i-1}, n_{i}\right)}=L / B_{n_{i-1}, n_{i}}
$$

$t_{C\left(n_{i-1}, n_{i}\right)}$, that is similar to $t_{0}$ depending on different routing protocols in network, is the routing table calculation and update time of node $n_{2}$. So, the Eq. 2 that computes the perception time for nodes to the network topology change can be written as:

$$
t_{i}=t_{i-1}+L / \text { Datarate }+L / B_{n_{i-1}, n_{i}}+t_{C\left(n_{i-1}, n_{i}\right)}
$$

By Eq. 5, every node in the network can be traversed by widely searching from two nodes where network topology changed, and the perception time of any nodes to this topology change can be computed.

\section{DRL Dynamic Routing Lookup Algorithm}

According to the hypothesis researched in this paper, the topology change information is disconnection of connection or recovery of disconnected connection. We can form complete network topology by three parts: the first two are static neighbor index vector and routing table that composed of cache, on this basis, the third is a topology change series that record every changed topology information and the corresponding time. Then we can complete the routing of each node based on its perception time when topology changes.

Firstly, we have the following conclusion proved:

Conclusion: In graph $G(V, E)$, ShortestPath $h_{n 1, n 2}=\left\{n_{1}, \cdots n_{2}\right\}$ is the shortest path from node $n_{1}$ to node $n_{2}$ with length $l$. If $m_{1}, m_{2} \in V$, the connection $\left(m_{1}, m_{2}\right) \in E$ and $\left(m_{1}, m_{2}\right) \notin$ ShortestPath $_{n 1, n 2}$, ShortestPath ${ }_{n 1, n 2}$ is still the shortest path from $n_{1}$ to $n_{2}$ when $\left(m_{1}, m_{2}\right)$ is failed in graph $G^{\prime}\left(V, E-\left\{m_{1}, m_{2}\right\}\right)$.

Proof: Using reductio ad absurdum.

Assumed that $\left(m_{1}, m_{2}\right)$ was failed, and the shortest path from $n_{1}$ to $n_{2}$ is ShortestPath ${ }_{n 1, n 2}{ }^{\prime}=\left\{n_{1}, \cdots n_{2}\right\}^{\prime} \neq$ ShortestPath $_{n 1, n 2}$ after updating in graph $G^{\prime}\left(V, E-\left\{m_{1}, m_{2}\right\}\right)$ of which the length is $l^{\prime} .\left(m_{1}, m_{2}\right) \notin$ ShortestPath $_{n 1, n 2}$, the length of ShortestPath $_{n 1, n 2} \in G^{\prime}\left(V, E-\left\{m_{1}, m_{2}\right\}\right)$ is $l$, ShortestPath ${ }_{n 1, n 2}$ ' and ShortestPath ${ }_{n 1, n 2}$ are paths from 
$n_{1}$ to $n_{2}$ in graph $G^{\prime}\left(V, E-\left\{m_{1}, m_{2}\right\}\right)$, and now the shortest path is ShortestPath ${ }_{n 1, n 2}{ }^{\prime}$, so we obtained that $l^{\prime}<l$.

Then, $\quad\left(m_{1}, m_{2}\right) \notin G^{\prime}\left(V, E-\left\{m_{1}, m_{2}\right\}\right) \quad, \quad$ so $\quad\left(m_{1}, m_{2}\right) \notin$ ShortestPath $_{n 1, n 2}{ }^{\prime} \quad, \quad$ and ShortestPath ${ }_{n 1, n 2}{ }^{\prime} \in G(V, E) . l<l^{\prime}$ will be obtained from ShortestPath ${ }_{n 1, n 2}$, ShortestPath ${ }_{n 1, n 2}{ }^{\prime}$ are the paths from $n_{1}$ to $n_{2}$ in graph $G^{\prime}\left(V, E-\left\{m_{1}, m_{2}\right\}\right)$.

Contradictorily! So the hypothesis is wrong, and ShortestPath ${ }_{n 1, n 2}$ is still the shortest path from $n_{1}$ to $n_{2}$ in graph $G^{\prime}\left(V, E-\left\{m_{1}, m_{2}\right\}\right)$.

According to the conclusion, while the connection failure or recovery inducing topology changes in the network, part of the routing information in the original network is still available. We need to create a network topology relationship series during the process of the topology changing, we can obtain a complete description of the network topology under the circumstance that the original routing tables are not completely changed. The principle of DRL dynamic routing algorithm is shown in Fig. 1.

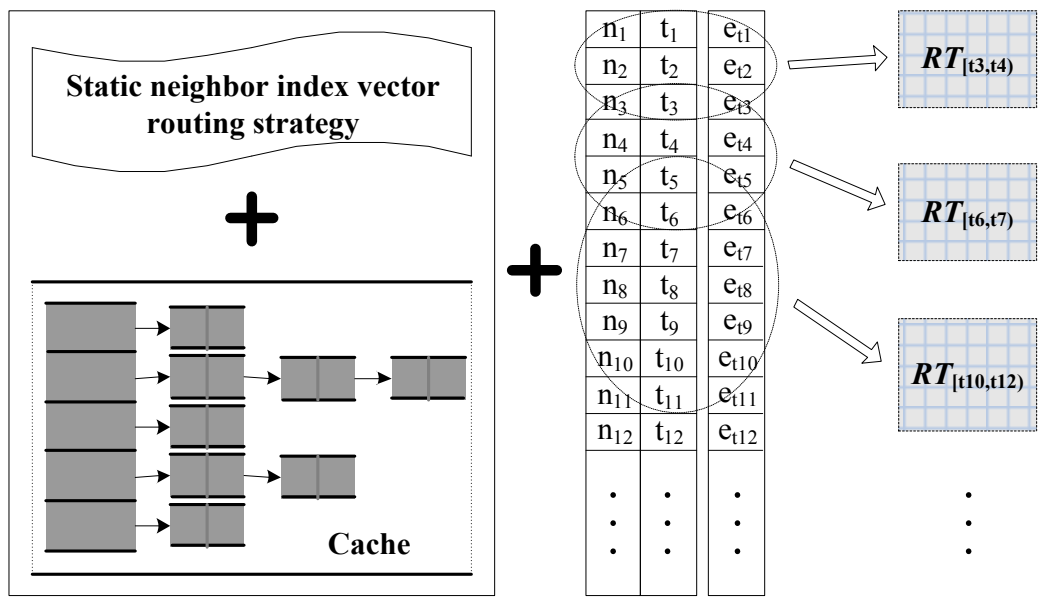

Figure 1. Dynamic routing strategy based on topology perception time series

So, by traversing network topology relation series we established, we obtain all the failure connections in the current network topology. The routing tables will not be immediately recalculated during the topology change, only the topological changing information is stored in the topology relation series. When packet routing is required, we conduct the current state of routing updates cooperate with the change of network topology series on the basis of the original static index vector routing strategy, that is to say, complete routing tables are established to entire network. As a result, the DRL dynamic routing algorithm designed shows in Table 1. 
Table 1. Dynamic routing algorithm based on topology perception time series

Pseudo code for dynamic routing algorithm for:

1 Calculate network routing table $R T_{t}$ according to the strategy using neighbor index vector,;

2 Add the topology change message to topological relation series $S$ when network topology changes;

3 Packet $p k t$ arrives;

4 Determine the shortest path $P$ ( $s r c$ to $d s t$ ) by traversing the routing table;

5 Calculate topology perception time $t$ based on formula $t_{i}=t_{i-1}+L /$ Datarate $+L / B_{n_{i-1}, n_{i}}+t_{C\left(n_{i-1}, n_{i}\right)}$;

6 Determine the current failure connection by corresponding topology changes series $S^{\prime}=\left\{\left(m_{1}, m_{2}\right) \mid \forall\left(m_{1}, m_{2}\right) \in S^{\prime}, t_{m 1, m 2} \in S \& t_{m 1, m 2}<t\right\} ;$

7 Judge: if $\left(m_{1}, m_{2}\right) \notin P$, perform 8 ; if $\left(m_{1}, m_{2}\right) \in P$, perform 11;

8 Determine the current complete network topology $G^{\prime}(V, E)$ based on $t$ and $S^{\prime}$;

9 Update routing table $R T_{t^{\prime}}$ by reusing strategy using static neighbor index vector;

10 Traverse route table $R T_{t^{\prime}}$ and determine the shortest path $P^{\prime}$, then perform 11;

11 Traverse all the nodes in the shortest path;

12 Determine the next hop NextHop of packet by extracting the previous $k=\left[\log _{2}^{M}\right\rceil$ of data packets $p k t$ as index;

13 Sending data to the next-hop router;

14 Judge: If NextHop $\neq d s t$, perform 11 again; else, perform 15;

15 End.

\section{Simulation Experiments and Results Analysis}

In order to validate the performance of the dynamic routing strategy, we realized the strategy based on $\mathrm{OMNeT}++$ simulator, and simulation tests are done for correctness and efficiency of dynamic routing algorithm. Experimental environments are: simulation host for dual core $3.2 \mathrm{GHz}$, 4G RAM, Ubuntu10.04.4 LTS operation system and $\mathrm{OMNeT}++4.2 .2$ version of the simulator. Network topology generated by using $\mathrm{OMNeT}++$ extension module ReaSE generator, experimental results were taken from the average of five results in the process of the simulation.

In order to test the simulation performance of dynamic routing strategy, memory usage and time consumption of the routing table were tested by setting up different size network topology and connection failure event. Performance of dynamic routing strategy in this paper was analyzed compared with strategy of flat and static neighbor index vector. In the simulation process, ReaSE topology generator divided network into two layers for the unit with the domain, whose inter-domain reflected the power-law distribution and intra-domain reflected randomness and generated a comprehensive, closer to the real network topology. Topology size expanded from 200 to 2000 and the node degree is set to 3.2 on average, every topology randomly generated $3000 \mathrm{TCP}$ connections and sent 1040bits data packet per connection. The experimental results for memory usage and simulation time consumption are shown in Fig.2 and Fig. 3. 


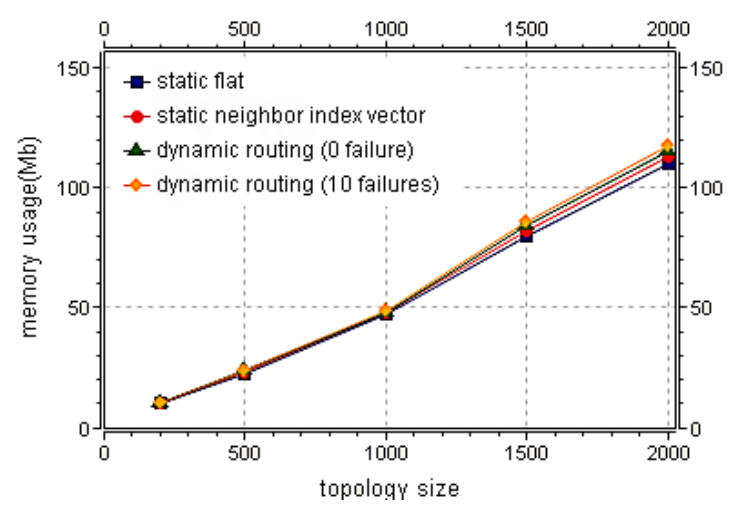

Figure 2. Different routing strategies storage space utilization

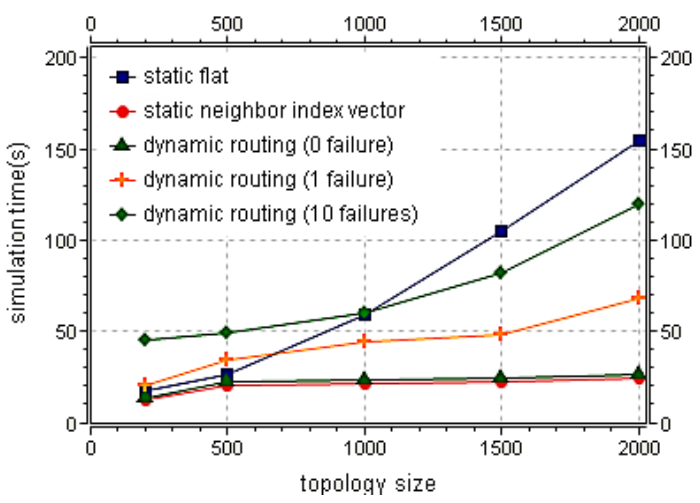

Figure 3. Different routing strategies simulation run time

As is shown in Fig. 2, each routing policy on the storage space almost has the same consumption, and little impact on the storage space for different sizes of connection failure. Because dynamic routing algorithm proposed in this paper only needs to record the topology change series of each routing node and perception time with small burden of storage space. In large-scale network simulation environment, this strategy enables dynamic perception for the network topology changes of each node and correctly routed without larger memory burden. Therefore, this method is more suitable for large-scale network simulation.

In Fig. 3, the simulation run time was compared under the failure conditions that different topologies and different sizes of connections were invalidation. Run time of static flat strategy simulation is much higher than static neighbor vector strategy and dynamic routing strategy while in the same topology and same simulation time, and the latter two remain at a low level. With the expansion of the failure scale, the simulation time increased significantly. There are two main reasons: first, due to the connection failure caused by topology change, the simulation kernel traverses each node in the network from location of connection failure, calculates and saves the overhead caused by each node; Secondly, when the route is looking up, there will be some time overhead to select a topology change series, compare with perception time and current time, then judge whether or not the shortest path contains the disconnected connections. But from the overall, the simulation running time of dynamic routing strategy is still low.

Through the analysis above, dynamic topology perception routing strategy based on time series can be more truly reflect the influence on routing calculation results caused by routing topology changes, and the simulation performance remains in a relative high level in case of the network topology and failed proportion expanding. 


\section{Summary}

Aimed to the poor authenticity of dynamic routing strategy and low performance in network simulation, this paper addressed the dynamic routing abstract model, and proposed the dynamic routing strategy based on topology perception time series. With perception time, we solved the simulation process time distortion problems in the dynamic routing calculation process. The series of topological change relation corresponding to perception time based on static routing table neighbor index vector formed a complete description of the entire network topology, and DRL dynamic routing lookup algorithm achieved routing lookup was designed to avoid unnecessary time-cost in routing calculation. Experimental results showed that the proposed routing strategy is closer to the actual situation and with high efficiency.

\section{References}

[1] La-yuan LI, Chun-lin LI. Dynamic routing protocol for computer networks with clustering topology[J]. Journal of Systems Engineering and Electronics, 2012,10(1): 44-53.

[2] Thorenoor S G. Dynamic routing protocol implementation decision between EIGRP, OSPF and RIP based on technical background using OPNET Modeler[C], Second International Conference on Computer and Network Technology. Bangkok, Thailand. IEEE.2010: 191 - 195

[3] Zhi-yu HAO, Xiao-chun YUN, Hong-li ZHANG. An efficient routing mechanism in network simulation[J]. Proceedings of the 20th ACM/IEEE/SCS Workshop on Principles of Advanced and Distributed Simulation. 2008. 84(10-11): 511-520.

[4] Yu-xin CHEN, Andrews J G. An upper bound on multihop transmission capacity with dynamic routing selection[J]. IEEE Transactions on Information Theory. 2012, 58(6):3751-3765.

[5] Arandjelovic R, Zisserman A. Extremely low bit-rate nearest neighbor search using a set compression tree[J]. IEEE Transactions on Pattern Analysis and Machine Intelligence, 2014, 36(12): 2396-2406.

[6] Riley G, Fujimoto R, Ammar M. Stateless routing in network simulations[C], Proceedings of the 8th International Symposium on Modeling, Analysis and Simulation of Computer and Telecommunication Systems. 2000, 524-531.

[7] Mishra V, Jangale S, Analysis and comparison of different network simulators[C], Special Issue for International Technological Conference- 2014.Vivekanand, India. Education Society's Institute Of Technology, 2014: 5. 